\title{
Is acupuncture no more than a placebo? Extensive discussion required about possible bias (Review)
}

\author{
SHIZHE DENG ${ }^{1 *}$, XIAOFENG ZHAO ${ }^{2 *}$, RONG DU $^{1}, \mathrm{SI} \mathrm{HE}^{1}$, YAN WEN $^{1}$, \\ LINGHUI HUANG $^{1}$, GUANG TIAN ${ }^{1}$, CHAO ZHANG $^{1}$, ZHIHONG MENG $^{1}$ and XUEMIN SHI ${ }^{1}$ \\ ${ }^{1}$ Department of Acupuncture and Moxibustion; ${ }^{2}$ Institute of Acupuncture and Moxibustion, \\ First Teaching Hospital of Tianjin University of Traditional Chinese Medicine, Tianjin 300193, P.R. China
}

Received August 25, 2014; Accepted June 16, 2015

DOI: $10.3892 / \mathrm{etm} .2015 .2653$

\begin{abstract}
Numerous randomized controlled trials (RCTs) of acupuncture have been conducted in recent years. The results of several studies implied that acupuncture was only a powerful placebo; however, certain studies demonstrated that verum acupuncture had a greater effect than placebo and the mechanisms between a verum acupuncture group and a placebo/sham group were different. Researchers attempted to investigate the inherent factors that may potentially influence the results of trials. Certain problems observed in acupuncture RCTs also occurred in RCTs in other fields, including insufficient sample size, high dropout rates, inadequate follow-up and randomization. The study of acupuncture is so complex that specific methodological challenges are raised, which are frequently overlooked, including sham interventions, blinding, powerful placebo effects (even stronger than an inert pill) and variations in acupuncture administration. The aforementioned problems may contribute to bias, and researchers systematically attempt to solve these problems. The present review aimed to suggest techniques to design high-quality studies, minimize the placebo effect and optimize acupuncture administration in acupuncture studies. If these problems are addressed, then the results of acupuncture studies may be different.
\end{abstract}

Correspondence to: Professor Zhihong Meng, Department of Acupuncture and Moxibustion, First Teaching Hospital of Tianjin University of Traditional Chinese Medicine, 314 West Anshan Avenue, Tianjin 300193, P.R. China

E-mail: profmengzhihong@163.com

${ }^{*}$ Contributed equally

Key words: acupuncture, randomized controlled trials, placebo effect, bias

\section{Contents}

1. Introduction

2. Problems with study design

3. A powerful placebo effect may result in a potential bias

4. Insufficient acupuncture administration

5. Conclusion

\section{Introduction}

Although acupuncture is a component of traditional Chinese medicine (TCM) that may be traced back $>2,500$ years in China, the interest of Western countries in acupuncture has increased in recent decades (1). Numerous clinical reports have suggested that acupuncture may be a reasonable option for a number of clinical conditions, including neural hearing loss (2), back pain (3) and certain injuries in athletes (4). The effectiveness of acupuncture remains controversial among researchers, with opinions from 'acupuncture is virtually useless' (5) to 'acupuncture deserves a respectful hearing' (6).

A number of clinical studies of acupuncture have been conducted since the application of evidence-based medicine to acupuncture. A good deal of evidence was collected with increasing numbers of RCTs; however, certain results were contradictory for the same condition (7). Furthermore, a previous study (8) indicated that there was no significant difference between verum (applied according to the principles of TCM) and sham acupuncture groups. Therefore, certain articles have implied that acupuncture is only a powerful placebo effect $(9,10)$.

Certain trials demonstrated that the mechanisms were different between the verum acupuncture and placebo/sham acupuncture groups. For instance, acupuncture analgesia and placebo analgesia had a different pattern of associated brain activation (11-13). Furthermore, previous studies on animal models evaluating the mechanisms of action of acupuncture demonstrated more positive results compared with studies conducted in humans (14).

Considering the aforementioned findings, it is valuable considering in depth the reasons for the contradictory or equivocal results that resembled random events in RCTs of acupuncture. Problems with study design; a powerful placebo 
effect from sham intervention and insufficient acupuncture treatment groups may contribute to the bias.

\section{Problems with study design}

Common problems. An article have discussed the design of acupuncture studies (14). Problems in acupuncture RCTs also occurred in RCTs in other fields; such problems include insufficient sample size, high dropout rates, inadequate follow-up and randomization. However, acupuncture studies are so complex that specific methodological challenges are raised, such as sham interventions and blinding procedures.

Randomization. Adequate randomization ensures that every subject has an equal opportunity to be allocated to the treatment or control groups, including suitable generation of an unpredictable randomized allocation sequence and sufficient allocation concealment (15). Larger estimates about study results were identified in non-randomized compared with randomized studies (16). Inadequate and unclear allocation concealment may overestimate treatment effects by 41 and $30 \%$, respectively (17). Suitable methods used to generate the allocation sequences are random-number tables or computer random-number generators; by contrast, inadequate methods include alternate assignment and assignment by odd/even birth date or hospital number $(15,17)$.

Allocation concealment is used to prevent bias from researchers that may influence the assignment of subjects until allocation. Methods such as opaque, sealed envelopes and central randomization were considered to be adequate in the process of allocation concealment (18). A previous review demonstrated that only $26 \%$ of included trials applied adequate sequence generation, and $29 \%$ applied adequate allocation concealment methods (19). Therefore, adequate randomization methods should be implemented and should also be clearly reported in RCTs. We suggest that future large multicenter RCTs should apply central randomization, in order to reduce the bias of RCTs.

Blinding. The blinding procedure is an essential part of RCTs, since it may reduce potential bias in the overall procedure (such as bias from the patients and evaluator). Previous studies $(17,20)$ demonstrated that non-blinded trials may overestimate the treatment effects by as much as $17 \%$. Investigators should clearly report the blinding procedure that was applied in their studies rather than describing the blinding procedure as double-blind (21).

Blinding procedures often include participant and therapist blinding. However, this is challenging in acupuncture RCTs, since the needle is manipulated by the acupuncturist and thus blinding of the acupuncturist is inappropriate (22). Blinding the patients and the evaluator is an alternative. In this process, a researcher may evaluate the blinding effect, not just report that the patients were blinded (23).

Researchers have implied that, unlike inexperienced subjects or subjects with limited acupuncture experience, experienced subjects may be able to distinguish the different appearance or sensation between real and sham acupuncture (24). For instance, a number of patients knew the exact sensation associated with acupuncture. Thus, subjects with different background knowledge and previous experience of acupuncture may induce a different result in the blinding procedure.

Other factors $(24,25)$ also influence the blinding procedure, including the needle point (a needle in an insensitive point may have a better blinding effect), the visual impact of needling (if the subject observed the manipulation of the acupuncturist, the effect of the blinding procedure may be influenced), and the therapists (the needling differences between acupuncture therapists should be minimized).

Control groups in acupuncture trials. The use of an appropriate control group was found to improve the reliability of acupuncture studies (26). However, selecting an ideal control group for acupuncture research is a challenging methodological issue (27). Questions about acupuncture cannot be answered by a single control group, since acupuncture is a complex intervention. Therefore, an appropriate control group should be selected according to the specific questions being asked in the study (28). The common categories of control groups in acupuncture studies are: Waiting-list control (no-treatment control group); standard care (such as medications); and sham interventions, which attempt to manage placebo and other nonspecific effects. The placebo effect cannot be managed in the waiting-list control and standard care groups and thus attention has been focussed on the control groups of sham interventions.

The terms 'sham acupuncture' and 'placebo needle' have not been clearly defined in clinical trials. In current research, the two terms are confusing: In certain acupuncture studies, the two terms were used to describe the non-penetrating sham needle $(29,30)$, whereas a review (31) described 'placebo needles' as non-penetrating sham needles and 'sham acupuncture' as needling at the wrong points or non-points.

Sham intervention is a complicated control which has been often applied as a control for nonspecific or placebo effects, in order to investigate whether verum acupuncture is more effective than sham intervention (28). The benefit of this control is that the blinding procedure may be more effective compared with other methods, and the disadvantages are the potential nonspecific needling effects (28). Therefore, partly because of the non-specific needling effects, the differences in clinical effects between the real and sham acupuncture groups are unclear.

Selecting an appropriate sham intervention. If a sham intervention was an absolutely inert procedure and its appearance was difficult to distinguish from the real acupuncture group, it could be regarded as the optimum condition in acupuncture RCTs for determining the effects of acupuncture interventions (32).

Several sham interventions, including superficial needling, needling in irrelevant true points, needling in non-points, non-penetrating sham needles, non-needling sham interventions and minimal acupuncture, were often used in acupuncture RCTs (28). Each type of sham intervention has certain advantages and disadvantages. All the aforementioned methods of sham intervention cannot be regarded as absolutely inert interventions, as they may induce certain therapeutic effects (33-36). In addition, the various manipulations and insert directions were not allowed by the device (37) in a non-penetrating sham needle. 
A

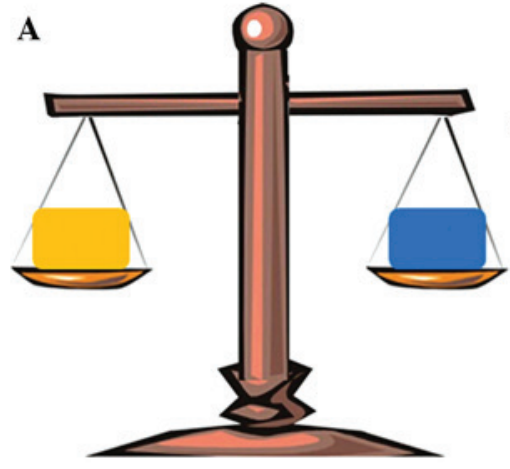

Acupuncture administration

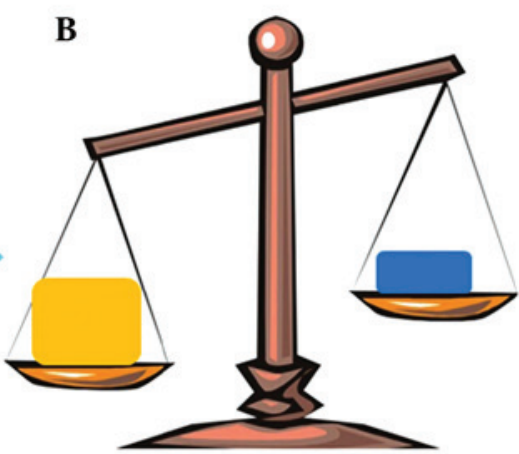

Placebo control group

Figure 1. Possible bias may influence the results of acupuncture studies. (A) Numerous results of acupuncture studies implied that acupuncture was only a powerful placebo. A high-quality study design, a minimized placebo effect and optimal acupuncture administration may contribute to reducing the potential bias. (B) If these problems are solved, the differences between acupuncture administration and placebo control groups may be significant.

This sham intervention-induced effect may result in the difference between verum and sham group not being significant (38). In addition, these controls were often investigated in inexperienced subjects $(29,39)$, so the blinding of this method should be considered when the subjects have had extensive experience in acupuncture.

Designing an appropriate control requires understanding the traditional acupuncture theory and the modern mechanism of acupuncture, and should match the specific objective of the study. To date, no sham intervention can be regarded as an ideal control and this question should be further investigated by methodologists.

\section{A powerful placebo effect may result in a potential bias}

Placebo effect. Positive results were often produced from studies that applied a non-acupuncture control group, and the results were usually negative in studies that used sham acupuncture or mock transcutaneous electrical nerve stimulation (40). Vickers et al (41) found that potent placebo effects were contributing to the total effects of acupuncture. Generally, placebo implied an inert substance or procedure and a placebo effect was an essential component of all therapeutic interventions (42). Intervention should be divided into specific and placebo (non-specific) aspects in order to design a placebo-controlled procedure (43).

Specific elements was considered to be responsible for the specific therapeutic actions, and other aspects that influenced the effects were placebo elements, such as the patient expectations and the communication between therapists and subjects (43). A previous study considered that the placebo effect was derived from factors included in a social, cultural and clinical context, rather than from the sham interventions per se $(44)$. Other studies $(42,45,46)$ demonstrated that a placebo effect was a complexity resulting from psychological, social and cultural background factors, as well as real physiological responses. Notably, one study (47) demonstrated that the placebo effect had a 'dose-dependent response': Although the intervention was sham, if people received more care, their outcome would be improved.
The psychological mechanisms (42) of the placebo effect include many aspects associated with the procedure of acupuncture, including expectations, memory, motivation, cognitive and conditioning mechanisms.

Minimizing the placebo effect. If the major mechanisms of placebo responsiveness could be identified, strategies aimed at minimizing placebo effects could be developed, so that the true effect of an intervention can be revealed in trials (48). The factors influencing placebo effects in acupuncture studies included frequent communication between acupuncturist and subject, the 'ritual' of acupuncture, the expectations of patients and certain potential physiological effects caused by the procedures of acupuncture (49).

Systematic research was conducted to explore how to minimize the placebo effect. Researchers successfully reduced the placebo effect by decreasing the interaction between therapists and participants (47). Other studies demonstrated that strong placebo effects could significantly influence the outcome of subjective symptoms and this significant influence may be confined to subjective outcomes (50). Therefore, in future experiments, multiple outcome measurements should be selected, including subjective and objective outcomes.

Although the placebo effect is complex, research demonstrated that the placebo effect from sham acupuncture was stronger compared with the effect from a placebo pill (50). These results may be due to more complex medical interventions having a higher placebo effect than medication (51).

Given the potential physiological effects of sham interventions and the powerful placebo effects, the results of acupuncture RCTs may be undervalued in numerous studies (52). Although sham acupuncture exerted a powerful placebo response and uncertain physiological mechanisms, evidence also demonstrated a specific effect from acupuncture which was better than the 'placebo effect' (53).

\section{Insufficient acupuncture administration}

Acupuncture administration. One of the least investigated areas of acupuncture research may be acupuncture administra- 
tion. Researchers have to increase the effectiveness of drugs to prove that their effect is greater than placebo control; however, this has not been the case in acupuncture trials. An insufficient acupuncture treatment group may also provide potential bias regarding study outcomes. The results of acupuncture efficacy based on insufficient acupuncture treatment groups can be compared with formulating conclusions on the pharmaceutical efficacy of a drug with an inadequate dose (54). It is important to emphasize that acupuncture is not a straightforward needling intervention. The evaluation is therefore far more challenging than that of a pharmacological agent.

When the results of two studies about acupuncture on hypertension are compared $(10,55)$, the data may demonstrate that the effects of active or verum acupuncture groups are different. Macklin et al (10) revealed that active acupuncture groups provided no greater benefit than sham acupuncture, while Flachskampf et al (55) reported a clear-cut effect on blood pressure in the active treatment group. The setting of acupuncture administration was different in the two trials. The acupuncture procedure in the trial by Flachskampf et al (55) was that patients received 22 sessions in 6 weeks, with 5 sessions/week conducted in the first 2 weeks and 3 sessions/week conducted in the following 4 weeks. Each session lasted $30 \mathrm{~min}$ after the needle was inserted into the points. The needling points were selected according to the Chinese type of hypertension in Traditional Chinese Medicine theory, and the acupuncture details, including angle, depth and type of manipulation of needling, were consistent with typical prescriptions. By contrast, in the trial that obtained a negative result (10), the principles of diagnoses and treatment were consistent with the description of Cheng (56). The treatment procedure consisted of $\leq 12$ sessions, generally twice-weekly, over 6-8 weeks with each session providing a 30-min treatment. Therefore, it can be concluded that the difference in the acupuncture administration setting may induce certain potential bias.

Principal features of acupuncture intervention efficacy. Although there were a variety of factors that may account for the improvement in the acupuncture group, two principal features should be emphasized: Acupoint specificity and manipulation of acupuncture.

Acupoint specificity. Acupoint specificity is often considered as the foundation of the theory and treatment of acupuncture (57). Evidence derived from brain imaging and biological studies demonstrated that the point specificity does exist (58-61).

Clinical research identified that acupuncture stimulation of different points on similar body regions in patients with migraine reduced pain and induced different levels of cerebral glucose metabolism in pain-related brain regions (62). We strongly suggest that the international classification of acupoints by the World Health Organization should be used and referenced in clinical trials. The optimal selection of acupoints requires expert knowledge and standard textbooks and the accurate location of acupoints should be strictly conducted.

Acupuncture manipulation. Apart from acupoint localization, acupuncture manipulation was also considered to be a pivotal potential modifier of acupuncture effects. Normally, needling manipulation refers to various manipulations of acupuncture to induce a needling sensation once a needle is inserted (63). The fundamental manipulation techniques can be divided into various aspects, and these techniques may be used alone or in combination, according to the clinical condition of the patient. Manipulation methods may differ in the depth of needling, the form of needle stimulation (e.g. manual vs. electrical), the intensity of stimulation and stimulus timing parameters (such as duration and frequency). Mastering the manipulations of acupuncture may improve its therapeutic effects in clinical practice, according to the theory of TCM.

Over the past several years, numerous studies have been published to investigate the effect of acupuncture manipulation. Previous studies $(64,65)$ have demonstrated that different acupuncture manipulations a different effect on pain, as demonstrated by cellular responses in mouse subcutaneous connective tissue. In addition, Han et al (66) revealed that different frequencies of electrical stimulation affected the release of different neuropeptides. Changes in functional magnetic resonance imaging also found that various durations of acupuncture intervention would influence the effect in the brain (67). Furthermore, Langevin et al (65) demonstrated that subtle differences in needle-manipulation techniques induced different cellular responses in mouse subcutaneous connective tissue and the maximal cellular responses were produced by specific combinations of cycle amplitude and cycle number.

Therefore, different acupuncture manipulations may play a role in the clinical outcomes and inappropriate acupuncture techniques may induce a potential bias in acupuncture studies. Due to the variations existing in acupuncture procedures, investigating the effect of acupuncture is more difficult than for other ordinary therapies. Currently, to the best of our knowledge, no generally accepted guidelines about how to perform an optimal acupuncture administration for each disease have been established. The details of acupuncture intervention were rarely objectively quantified in the methods of study, hence comparing or repeating studies was challenging (68). Therefore, it was emphasized that objectively quantified acupuncture administration should be applied and clearly reported in acupuncture research.

\section{Conclusion}

Acupuncture research should be conducted under strict quality control, as are other studies. Problems in the design of trials, including randomization, blinding and adequate control groups, should be considered and the following two treatment parameters should be explored: How to optimize the efficacy of acupuncture intervention, and how to minimize the impact of placebo effects. These problems are urgent issues faced by the acupuncture community.

Prior to conducting acupuncture RCTs, several of the aforementioned problems must be re-evaluated. A high-quality study design, a minimized placebo effect and optimal acupuncture administration would contribute to the reduction of the potential bias in acupuncture studies (Fig. 1).

\section{Acknowledgements}

This study was financially supported by a grant from the National Basic Research Program of China (973 Program; grant no. 2010CB530506). 


\section{References}

1. Marwick C: Acceptance of some acupuncture applications. JAMA 278: 1725-1727, 1997.

2. Rose DE: Evaluating acupuncture. Science 186: 196, 1974.

3. Cheung F: Modern TCM: Enter the clinic. Nature 480 (Suppl): 94-95, 2011.

4. Watts J: Feature: Olympian pins and needles. Lancet 366 (Suppl 1): 62-63, 2005.

5. Taub A: Thumbs down on acupuncture. Science 279: 159, 1998.

6. Kaptchuk TJ: Acupuncture: Theory, efficacy and practice. Ann Intern Med 136: 374-383, 2002.

7. Ernst E: Complementary and alternative medicine: Between evidence and absurdity. Perspect Biol Med 52: 289-303, 2009.

8. Haake M, Müller HH, Schade-Brittinger C, et al: German acupuncture trials (GERAC) for chronic low back pain: Randomized, multicenter, blinded, parallel-group trial with 3 groups. Arch Intern Med 167: 1892-1898, 2007.

9. Ernst E: Acupuncture-a critical analysis. J Intern Med 259: 125-137, 2006.

10. Macklin EA, Wayne PM, Kalish LA, et al: Stop hypertension with the acupuncture research program (SHARP): Results of a randomized, controlled clinical trial. Hypertension 48: 838-845, 2006.

11. Kong J, Kaptchuk TJ, Polich G, et al: Expectancy and treatment interactions: A dissociation between acupuncture analgesia and expectancy evoked placebo analgesia. Neuroimage 45: 940-949, 2009.

12. Kong J, Kaptchuk TJ, Polich G, et al: An fMRI study on the interaction and dissociation between expectation of pain relief and acupuncture treatment. Neuroimage 47: 1066-1076, 2009.

13. Harris RE, Zubieta JK, Scott DJ, Napadow V, Gracely RH and Clauw DJ: Traditional Chinese acupuncture and placebo (sham) acupuncture are differentiated by their effects on mu-opioid receptors (MORs). Neuroimage 47: 1077-1085, 2009.

14. Langevin HM, Hammerschlag R, Lao L, Napadow V, Schnyer RN and Sherman KJ: Controversies in acupuncture research: Selection of controls and outcome measures in acupuncture clinical trials. J Altern Complement Med 12: 943-953, 2006.

15. Schulz KF and Grimes DA: Generation of allocation sequences in randomised trials: Chance, not choice. Lancet 359: 515-519, 2002.

16. Carroll D, Tramèr M, McQuay $\mathrm{H}$, Nye $\mathrm{B}$ and Moore $\mathrm{A}$ : Randomization is important in studies with pain outcomes: Systematic review of transcutaneous electrical nerve stimulation in acute postoperative pain. Br J Anaesth 77: 798-803, 1996.

17. Schulz KF, Chalmers I, Hayes RJ and Altman DG: Empirical evidence of bias. Dimensions of methodological quality associated with estimates of treatment effects in controlled trials. JAMA 273: 408-412, 1995.

18. Zheng H,Li Y and Chen M: Evidence based acupuncture practice recommendations for peripheral facial paralysis. Am J Chin Med 37: 35-43, 2009.

19. Savović J, Jones HE, Altman DG, et al: Influence of reported study design characteristics on intervention effect estimates from randomized, controlled trials. Ann Intern Med 157: 429-438, 2012.

20. Wood L, Egger M, Gluud LL, et al: Empirical evidence of bias in treatment effect estimates in controlled trials with different interventions and outcomes: Meta-epidemiological study. BMJ 336: 601-605, 2008

21. Schulz KF and Grimes DA: Blinding in randomised trials: Hiding who got what. Lancet 359: 696-700, 2002.

22. Witt CM, Aickin M, Baca T, et al: Effectiveness Guidance Document (EGD) for acupuncture research-a consensus document for conducting trials. BMC Complement Altern Med 12: 148, 2012.

23. Birch S: Clinical research on acupuncture. Part 2. Controlled clinical trials, an overview of their methods. J Altern Complement Med 10: 481-498, 2004.

24. Tsukayama H, Yamashita H, Kimura T and Otsuki K: Factors that influence the applicability of sham needle in acupuncture trials: Two randomized, single-blind, crossover trials with acupuncture-experienced subjects. Clin J Pain 22: 346-349, 2006

25. Enblom A, Hammar M, Steineck G and Börjeson S: Can individuals identify if needling was performed with an acupuncture needle or a non-penetrating sham needle? Complement Ther Med 16: 288-294, 2008
26. Hróbjartsson A and Gøtzsche PC: Placebo interventions for all clinical conditions. Cochrane Database Syst Rev CD003974, 2010.

27. White P, Lewith G, Hopwood V and Prescott P: The placebo needle, is it a valid and convincing placebo for use in acupuncture trials? A randomised, single-blind, cross-over pilot trial. Pain 106: 401-409, 2003.

28. Moffet HH: Sham acupuncture may be as efficacious as true acupuncture: A systematic review of clinical trials. J Altern Complement Med 15: 213-216, 2009.

29. Streitberger K and Kleinhenz J: Introducing a placebo needle into acupuncture research. Lancet 352: 364-365, 1998.

30. Chae Y, Um SI, Yi SH, et al: Comparison of biomechanical properties between acupuncture and non-penetrating sham needle. Complement Ther Med 19 (Suppl 1): 8-12, 2011.

31. Berman BM: Seminal studies in acupuncture research. J Altern Complement Med 7 (Suppl 1): 129-137, 2001.

32. McManus CA, Schnyer RN, Kong J, et al: Sham acupuncture devices-practical advice for researchers. Acupunct Med 25: 36-40, 2007.

33. Langevin HM, Konofagou EE, Badger GJ, et al: Tissue displacements during acupuncture using ultrasound elastography techniques. Ultrasound Med Biol 30: 1173-1183, 2004.

34. Linde K, Streng A, Jürgens S, et al: Acupuncture for patients with migraine: A randomized controlled trial. JAMA 293: 2118-2125, 2005.

35. Diener HC, Kronfeld K, Boewing G, et al: Efficacy of acupuncture for the prophylaxis of migraine: A multicentre randomised controlled clinical trial. Lancet Neurol 5: 310-316, 2006.

36. Manheimer E: Selecting a control for in vitro fertilization and acupuncture randomized controlled trials (RCTs): How sham controls may unnecessarily complicate the RCT evidence base. Fertil Steril 95: 2456-2461, 2011.

37. Kaptchuk TJ: Placebo needle for acupuncture. Lancet 352: 992 , 1998.

38. Lundeberg T, Lund I, Näslund J and Thomas M: The Emperors sham-wrong assumption that sham needling is sham. Acupunct Med 26: 239-242, 2008.

39. Goddard G, Shen Y, Steele B and Springer N: A controlled trial of placebo versus real acupuncture. J Pain 6: 237-242, 2005.

40. Itoh K, Hirota S, Katsumi Y, Ochi H and Kitakoji H: Trigger point acupuncture for treatment of knee osteoarthritis - a preliminary RCT for a pragmatic trial. Acupunct Med 26: 17-26, 2008.

41. Vickers AJ, Cronin AM, Maschino AC, Lewith G, MacPherson H, Foster NE, Sherman KJ, Witt CM and Linde K; Acupuncture Trialists' Collaboration: Acupuncture for chronic pain: Individual patient data meta-analysis. Arch Intern Med 172: 1444-1453, 2012.

42. Finniss DG, Kaptchuk TJ, Miller F and Benedetti F: Biological, clinical and ethical advances of placebo effects. Lancet 375 : 686-695, 2010

43. Paterson C and Dieppe P: Characteristic and incidental (placebo) effects in complex interventions such as acupuncture. BMJ 330: 1202-1205, 2005.

44. Barrett B, Brown R, Rakel D, et al: Placebo effects and the common cold: A randomized controlled trial. Ann Fam Med 9: 312-322, 2011.

45. Bertisch SM, Legedza AR, Phillips RS, et al: The impact of psychological factors on placebo responses in a randomized controlled trial comparing sham device to dummy pill. J Eval Clin Pract 15: 14-19, 2009.

46. Wager TD, Rilling JK, Smith EE, et al: Placebo-induced changes in FMRI in the anticipation and experience of pain. Science 303: 1162-1167, 2004.

47. Kaptchuk TJ, Kelley JM, Conboy LA, et al: Components of placebo effect: Randomised controlled trial in patients with irritable bowel syndrome. BMJ 336: 999-1003, 2008.

48. Enck P, Benedetti F and Schedlowski M: New insights into the placebo and nocebo responses. Neuron 59: 195-206, 2008

49. Kaptchuk TJ: The placebo effect in alternative medicine: Can the performance of a healing ritual have clinical significance? Ann Intern Med 136: 817-825, 2002

50. Kaptchuk TJ, Stason WB, Davis RB, et al: Sham device v inert pill: Randomised controlled trial of two placebo treatments. BMJ 332: 391-397, 2006.

51. Kaptchuk TJ, Goldman P, Stone DA and Stason WB: Do medical devices have enhanced placebo effects? J Clin Epidemiol 53: 786-792, 2000

52. Kaptchuk TJ, Chen KJ and Song J: Recent clinical trials of acupuncture in the West: Responses from the practitioners. Chin J Integr Med 16: 197-203, 2010. 
53. Hopton A and MacPherson H: Acupuncture for chronic pain: Is acupuncture more than an effective placebo? A systematic review of pooled data from meta-analyses. Pain Pract 10: 94-102, 2010.

54. Berman BM: Clinical applications of acupuncture: An overview of the evidence. J Altern Complement Med 7 (Suppl 1): 111-118, 2001.

55. Flachskampf FA, Gallasch J, Gefeller O, et al: Randomized trial of acupuncture to lower blood pressure. Circulation 115: 3121-3129, 2007.

56. Cheng X (ed): Chinese Acupuncture and Moxibustion. Revised edition. Foreign Language Press, Beijing, 1999.

57. Rong PJ, Zhao JJ, Gao JH, et al: Progress of research on specificity of meridian acupoint efficacy. Chin J Integr Med 19: 889-893, 2013

58. Xing JJ, Zeng BY, Li J, Zhuang Y and Liang FR: Acupuncture point specificity. Int Rev Neurobiol 111: 49-65, 2013.

59. Na BJ, Jahng GH, Park SU, et al: An fMRI study of neuronal specificity of an acupoint: Electroacupuncture stimulation of Yanglingquan (GB34) and its sham point. Neurosci Lett 464: 1-5, 2009.

60. Bai L, Yan H, Li L, et al: Neural specificity of acupuncture stimulation at pericardium 6: Evidence from an FMRI study. J Magn Reson Imaging 31: 71-77, 2010.
61. Ren Y, Bai L, Feng Y, Tian J and Li K: Investigation of acupoint specificity by functional connectivity analysis based on graph theory. Neurosci Lett 482: 95-100, 2010.

62. Yang J, Zeng F, Feng Y, et al: A PET-CT study on the specificity of acupoints through acupuncture treatment in migraine patients. BMC Complement Altern Med 12: 123, 2012.

63. Huang T, Zhang W, Jia S, et al: A transcontinental pilot study for acupuncture lifting-thrusting and twisting-rotating manipulations. Evid Based Complement Alternat Med 2012: 157989, 2012.

64. Choi YJ, Lee JE, Moon WK and Cho SH: Does the effect of acupuncture depend on needling sensation and manipulation? Complement Ther Med 21: 207-214, 2013.

65. Langevin HM, Bouffard NA, Churchill DL and Badger GJ: Connective tissue fibroblast response to acupuncture: Dose-dependent effect of bidirectional needle rotation. J Altern Complement Med 13: 355-360, 2007.

66. Han JS, Chen XH, Sun SL, et al: Effect of low- and high-frequency TENS on Met-enkephalin-Arg-Phe and dynorphin A immunoreactivity in human lumbar CSF. Pain 47: 295-298, 1991.

67. Li K, Shan B, Xu J, et al: Changes in FMRI in the human brain related to different durations of manual acupuncture needling. J Altern Complement Med 12: 615-623, 2006.

68. Lewith GT, White PJ and Kaptchuk TJ: Developing a research strategy for acupuncture. Clin J Pain 22: 632-638, 2006. 